\title{
Assessment of Gearbox Fault DetectionUsing Vibration Signal Analysis and Acoustic Emission Technique
}

\author{
Vimal Saxena ${ }^{1}$ Nilendu Kar Chowdhury ${ }^{2}$ S. Devendiran ${ }^{3}$ \\ ${ }^{I} B$-tech, School of Mechanical and Building Science, VIT University, India \\ ${ }^{2} B$-tech, School of Mechanical and Building Science, VIT University, India \\ ${ }^{3}$ Asst. Professor, School of Mechanical and Building Science, VIT University, India
}

\begin{abstract}
Maintenance is a set of organised activities that are carried out in order to keep an item in its best operational condition with minimum cost acquired. Predictive maintenance $(P d M)$ is one of the maintenance program that recommends maintenance decisions based on the information collected through condition monitoring techniques, statistical process control or equipment performance for the purpose of early detection and elimination of equipment defects that could lead to unplanned downtime of machinery or unnecessary expenditures. Particularly Gears and rolling element bearings are critical elements in rotating machinery, so predictive maintenance is often applied to them. Fault signals of gearboxes or rolling-element bearings are nonstationary. This paper concludes with a brief discussion on current practices of PDM methodologies such as vibration analysis and Acoustic Emission analysis, which are widely used as they offers a complimentary tool for health monitoring or assessment of gears in rotating machineries.
\end{abstract}

Keywords:Acoustic emission, Data mining, Signal processing, Vibration Analysis

\section{INTRODUCTION}

The ever growing need to condition monitor a gear box, which plays a significant role as a machine element and finds its usage in wide variety of domestic and industrial application, especially in power transmission system, has promoted research in designing and implementing a rapid and accurate valuation of non-destructive techniques so as to prevent performance degradation of machinery or even catastrophic failure of the same. It is a well noticed fact that fault in a machine can be well noticed by the kind of noise it makes and same can be detected from its vibration. The ability to monitor the condition of machines in operating condition is one of major breakthrough in this area of research which is referred as "predictive" maintenance. The method can vitalize continuously operating plant such as the power generating and chemical industries, and can also be of considerable use in development of various machines. The present paper is a general discussion of the different analysis techniques which have been and can be applied in vibration signature analysis as well as in acoustic emission.

\section{TYPES OF DEFECT}

Gears and gearboxes are generally substantially made for high performance and reliability. However, problems do occur, and many are caused by static as well as dynamic reasons. Static causes which comprises of manufacturing defects and setting up errors, which can be observed even when the object is still whereas dynamic errors are those which can only be detected if either the object or the viewpoint is moving. Vibration signature analysis and Acoustic emission are the most popular techniques for gear fault diagnostics. In a machine under operation, vibration is always present. The levels of vibration usually increase with deterioration in the condition of the machine.

Table 1: Gear Failure Mechanisms

\begin{tabular}{|l|l|c|l|}
\hline $\begin{array}{l}\text { Failure } \\
\text { Mechanisms }\end{array}$ & Reason for damage & Name of the process & Result \\
\hline $\begin{array}{l}\text { Mechanical } \\
\text { damage }\end{array}$ & $\begin{array}{l}\text { Permanent indentation creating } \\
\text { gear meshing }\end{array}$ & Brinelling & Indentation \\
\hline Crack damage & $\begin{array}{l}\text { Manufacturing defect orstress } \\
\text { due to overload }\end{array}$ & Crack & Loss of dimensioning \\
\hline Wear damage & Gradual deterioration & Scuffing & - \\
\hline $\begin{array}{l}\text { Insufficient } \\
\text { lubrication and } \\
\text { contamination }\end{array}$ & Friction & - & Accelerate scuffing \\
\hline
\end{tabular}




\begin{tabular}{|c|l|c|l|}
\hline Incorrect design & $\begin{array}{l}\text { Poor choice of bearing type or } \\
\text { sizefor required operation. }\end{array}$ & $\begin{array}{l}\text { Low load carrying capacity } \\
\text { or low speed rating }\end{array}$ \\
\hline $\begin{array}{l}\text { Thermal } \\
\text { instability }\end{array}$ & $\begin{array}{l}\text { Large temperature difference } \\
\text { builds up }\end{array}$ & $\begin{array}{l}\text { It causes the bearings to } \\
\text { lose internal clearance and } \\
\text { become pre-loaded which } \\
\text { results in increase in heat } \\
\text { generation. }\end{array}$ \\
\hline Misalignment & $\begin{array}{l}\text { Manufacturing or setting up } \\
\text { errors, elastic deflection of } \\
\text { component under load, thermal } \\
\text { expansion }\end{array}$ & $\begin{array}{l}\text { Leads to premature pitting, } \\
\text { cage wear and finally } \\
\text { failure. }\end{array}$ \\
\hline Fatigue damage & $\begin{array}{l}\text { Material fatigue after } \\
\text { certain running }\end{array}$ & $\begin{array}{l}\text { Formation of minute crack } \\
\text { propagated }\end{array}$ \\
\hline $\begin{array}{l}\text { Faulty } \\
\text { installation }\end{array}$ & $\begin{array}{l}\text { Excessive pre loading and } \\
\text { misalignment }\end{array}$ & Noise running \\
\hline
\end{tabular}

The above defects are common in rotating gears. Vibration and acoustic emission are two important parameters which must be taken into consideration while examining a machine or its parts for defects. Both the techniques have their own importance in different working conditions. Various sensors records vibration signatures and acoustic emissions [1].

\section{FAult Detection TeChNiQues}

There are several techniques that can be employed to predict the condition of gears; these includevibration monitoring, acoustic emission, oil analysis, etc.

\begin{tabular}{|l|c|c|c|c|}
\hline Methods of analysis & $\begin{array}{c}\text { Acoustic } \\
\text { emission }\end{array}$ & $\begin{array}{c}\text { Oil } \\
\text { Analysis }\end{array}$ & Vibration & SPM meter \\
\hline Misalignment & & & $\mathrm{X}$ & \\
\hline Gear damage & $\mathrm{X}$ & $\mathrm{X}$ & $\mathrm{X}$ & \\
\hline Mechanical looseness & $\mathrm{X}$ & & $\mathrm{X}$ & \\
\hline Mechanical rubbing & $\mathrm{X}$ & & $\mathrm{X}$ & $\mathrm{X}$ \\
\hline Noise & $\mathrm{X}$ & $\mathrm{X}$ & \\
\hline Cracking & $\mathrm{X}$ & & & \\
\hline Minute gear damage & $\mathrm{X}$ & & & \\
\hline Minute Cracking & $\mathrm{X}$ & & & \\
\hline
\end{tabular}

\section{Vibrational Analysis}

The vibrational signals so obtained should first be converted into an electrical signal by a suitable transducer. Transducers are of mainly three types each of which measures velocity, relative displacement, and acceleration respectively. Acceleration transducers namely "accelerometers" are preferred over the other two as they have a very wide frequency range and dynamic range. There show a great deal of physical stability as they are very rugged and have no moving parts. With very small mass and physical dimension the accelerometer aces the other two methods.

The high impedance accelerometers can be minimized by using by using transistorized preamplifiers which convert from high impedance to low impedance and which at the same time can be used for signal conditioning such as amplification and integration.

The complex vibration signals, composed of mixtures of sinusoidal waveform of different waveform of different amplitudes, frequencies and phase differences can be broken down to vibration signals onto frequency components as shown in fig 1 .

The process of breaking down of vibration signals into individual frequency component is called frequency analysis. Time domain consists of amplitude that varies with time and frequency is where the magnitude varies with frequency. 

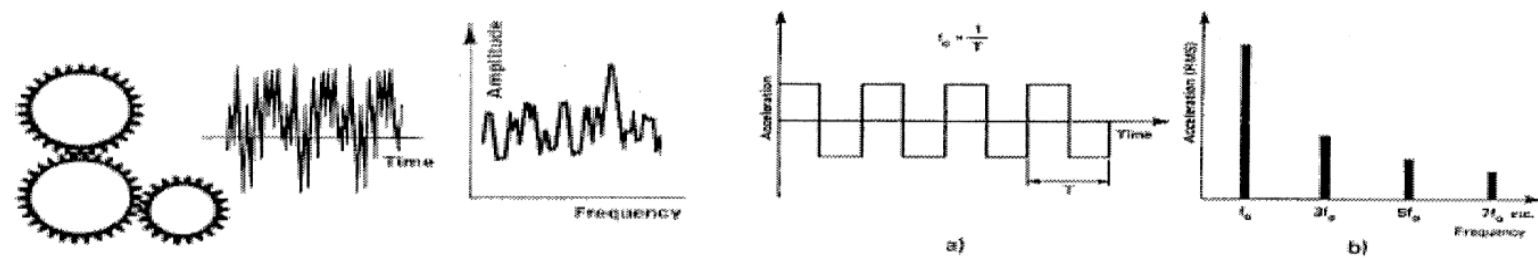

Fig 1: Typical vibration signals in time and frequency domains (Bruel\&Kjaer2)

Once the signal is acquired by the accelerometer, it gives a voltage reading that corresponds to the level of vibration. The analog signals given by the accelerometer are then collected by the Data Acquisition Card and transform them into digital signals so that it can read by an analyzing interface. The analyzing interface (computer software) is used to perform and use the analysis methods [3].

The figure 2 shown below shows the various steps involved in the vibration signature analysis of a sample and the synchronized order of its principle of working.

\section{FLOW CHART OF VIBRATION SIGNATURE ANALYSIS}

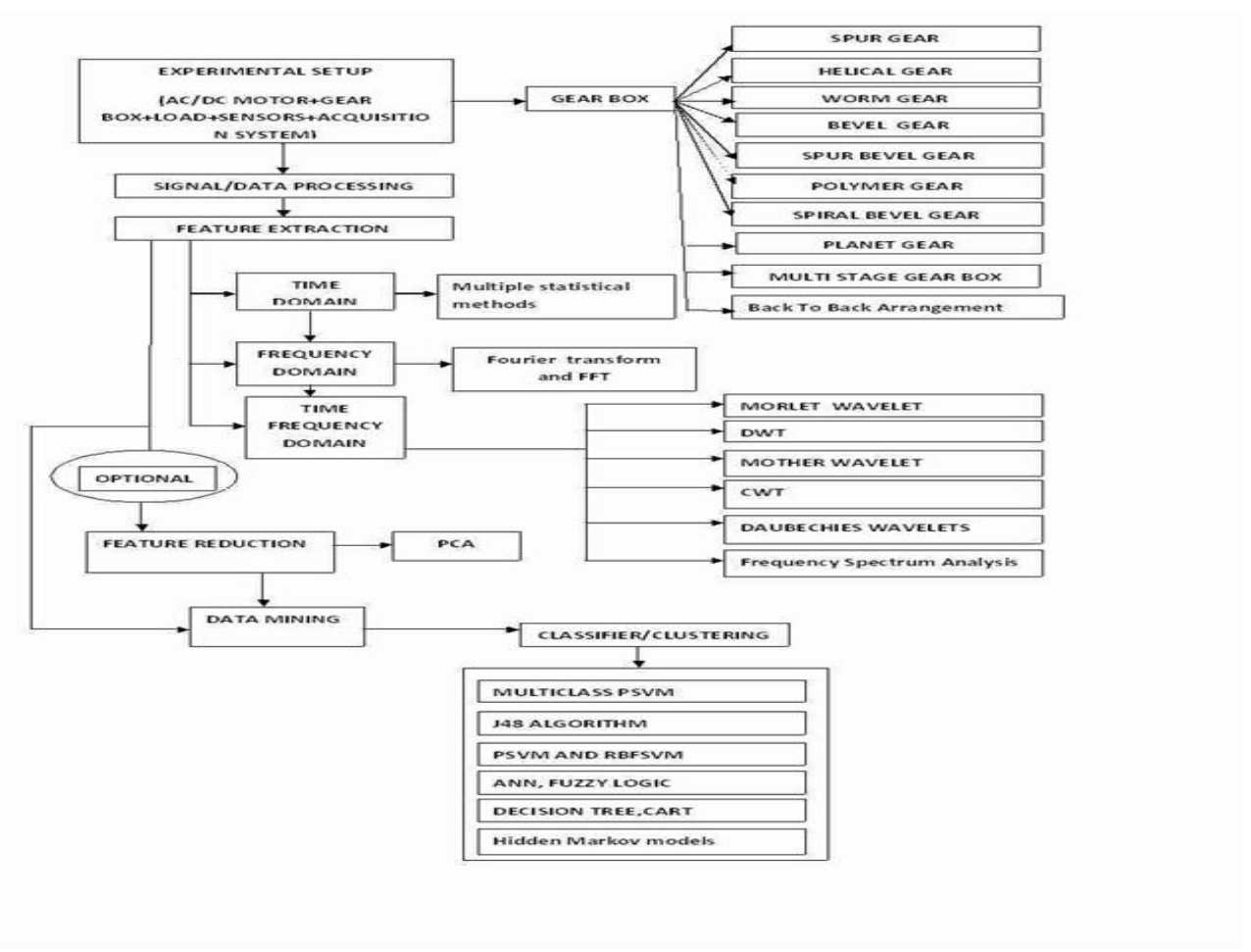

Fig.2Vibration signature analysis

IV. ACOUSTIC EMISSION TECHNIQUE:

Acoustic Emission(AE) is defined as the class of phenomenon whereby transient elastic waves are generated by the rapid release of energy from localized sources like places transient relaxation of stress and strain fields. Fracture, plastic deformation crack initiation and growth are a few examples of the phenomenon resulting in $\mathrm{AE}$. This dynamic nature of $\mathrm{AE}$ makes it highly potential technique for monitoring the integrity of critical structures and components.

First step of $\mathrm{AE}$ is to collect the signals from the gears. The sensing element for collecting the signals will be AE sensor. The analog signals so obtained are then collected by the Data Acquisition Card and transform them into digital signals so that it can read by an analyzing interface. The analyzing interface (computer software) is used to perform and use the analysis methods[4].

The following fig. 3 shows us the various steps in ordered fashion to be followed during the acoustic emission analysis of samples. 


\section{Flow Chart of ACOUSTIC Emission TechniQue}

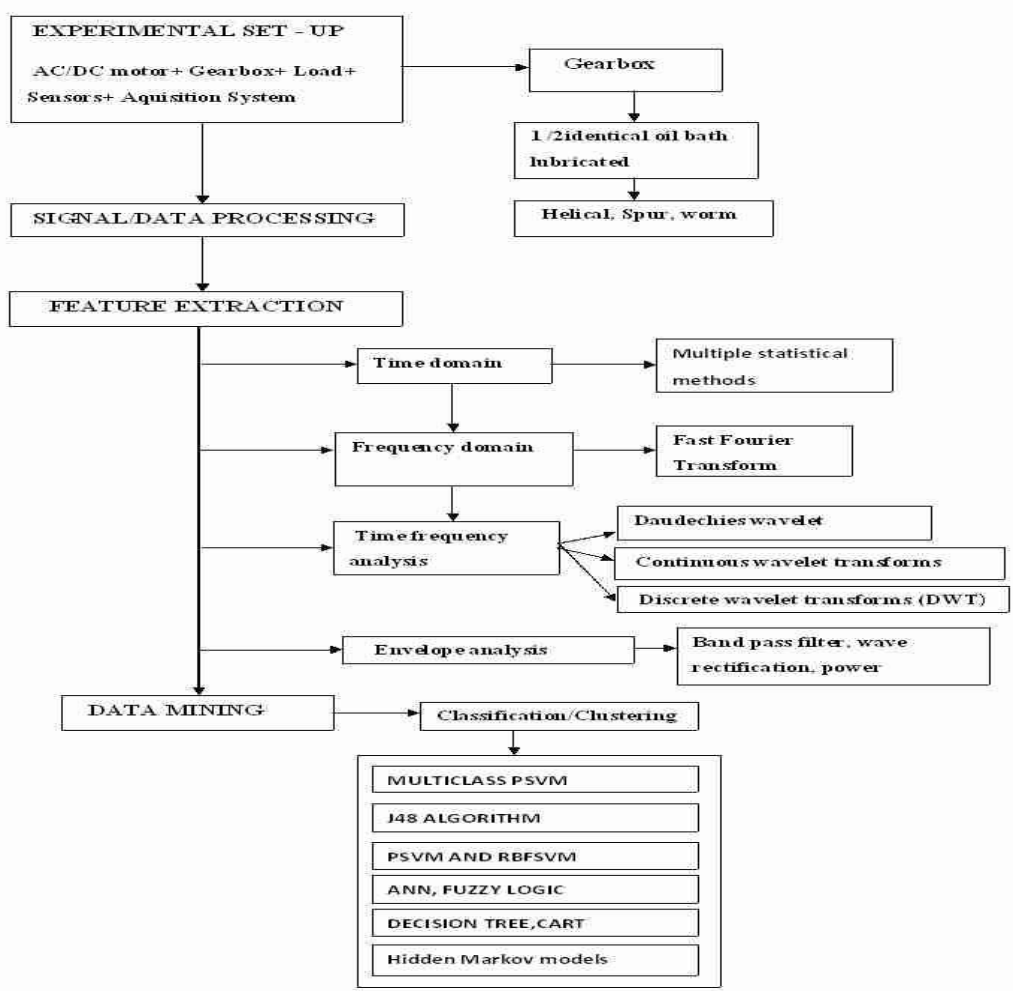

Fig. 3 Acoustic Emission technique

The process flow of the two techniques mentioned above can be summarized as follows:

\section{GENERAL SETUP USED}

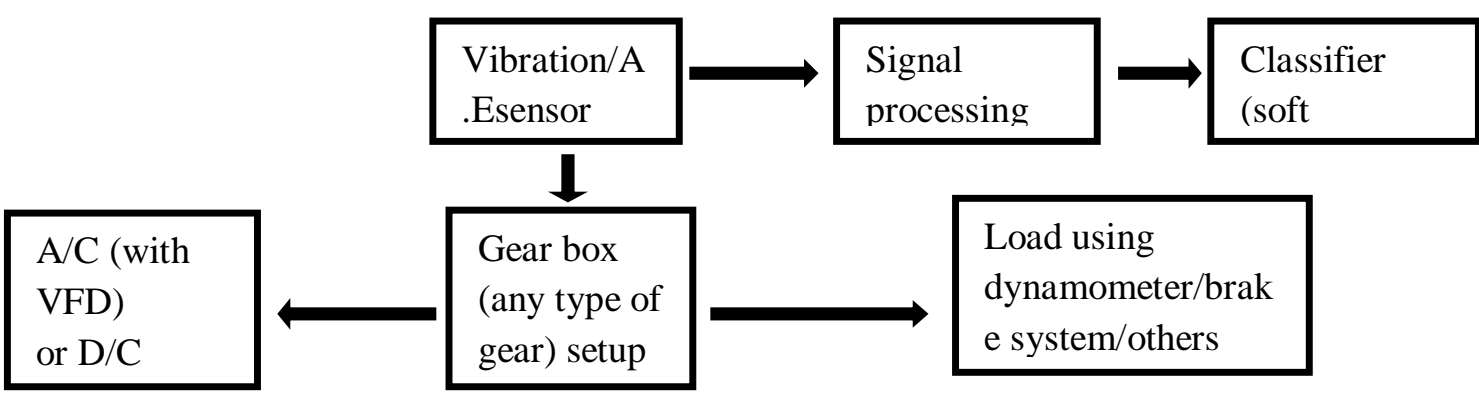

V. Analog To digital Conversion:

The process of converting analog signal (time domain) into digital form for further processing of the acquired signal can be achieved by microprocessors and works in power of two, refer to as binary numbers. A 12-bit ADC processor provides $4096\left(2^{12}\right)$ and a 16-bit ADC processor has a 65536 discrete intervals. The analog wave form is sampled at a fixed time interval and reconstructed to produce a digitized waveform. This ability of reproducing the original waveform totally depends on the spacing between the time interval and the number of samples points per unit time. This process is called sampling rate of sampling frequency.

Nyquist sampling theory states that in ADC conversion, the sampling must be twice the highest frequency component of interest. Fig 2 shows an example of undersampling (aliasing), which can be defined as a phenomenon which arises when sampling rate is less than double the wave frequency. With four sample points in $3 \mathrm{~ms}$, a wave of lower frequency (represented as dotted lines) appears and is not a valid representation of the signals. This can be corrected by allowing the low frequency signals to pass and blocking the high frequency signals. This is achieved by fixing an anti-aliasing filter. 


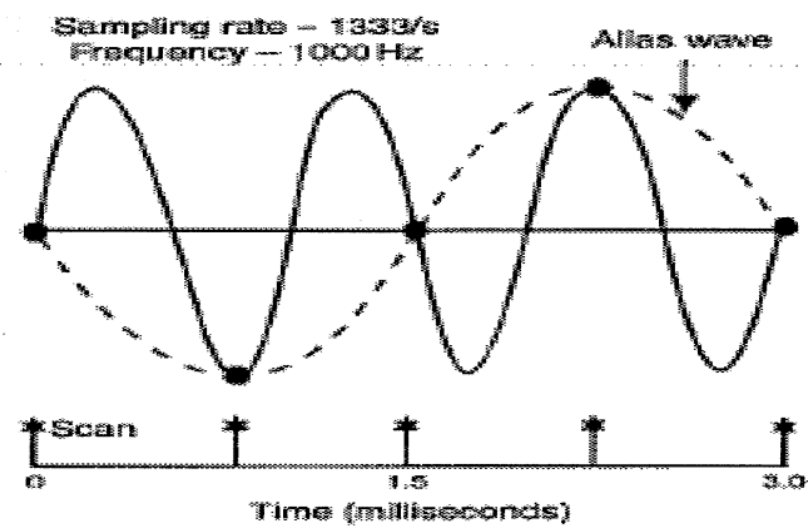

Fig 4: An example of aliasing (undersampling) [5]

\section{Signal Processing}

Signal processing is the enabling technology for the generation, transformation, andinterpretation of information and it's a very promising tool for the researchers in the field of non-destructive techniques. It comprises the theory, algorithms, architecture, implementation, and applications related to processing information contained in many different formats broadlydesignated as signals. It is the mathematical manipulation of an information signal to modify or improve it in some way. It is characterized by the representation of discrete time, discrete frequency, or other discrete domain signals by a sequence of numbers or symbols and the processing of these signals.

\section{Signal Processing Techniques:}

- Time Domain Techniques

- Frequency Domain Technique

- Time Frequency Analysis

- Envelope Analysis

\subsection{Time Domain TechniQues}

Time domain is the analysis of different parameters with respect to time. These parameters may include mathematical functions, physical signals, or time series of economic and environmental data. Commonly used tool to visualize real world signals in the time domain is oscilloscope.

For the analysis of recorded vibration and acoustic emission, time domain technique is one of the simpler approaches to detect and diagnose the same. There are $\mathrm{n}$ numbers of statistical parameters associated with the time domain technique. Some of which are RMS, Peak, Crest factor, Kurtosis,Clearancefactor, Impulse factor.

Root MEAN SQUaRe VALUe(RMS): The RMS value is computed by taking into account the time history of wave.It is a measure of the energy content in the vibration signature and hence is one of the most relevant statistical parameter.

$$
\text { - } \quad R M S=\sqrt{\frac{1}{n} \sum_{n} x^{2}(t)}
$$

Peak(Maximum Value):The peak value of signal is one of the important features for diagnosis

The value indicates the maximum value without any consideration of the time history of the wave.

$$
\text { - } \quad \text { Peak }=\frac{1}{2}(\max (x(t))-\min (x(t)))
$$

CREST FACTOR:The crest factor also sometimes called the 'peak-to-rms' ratio is defined as the ratio of peak value of a waveform to its RMS value.

$$
\text { - } \quad \text { Crestfactor }=\frac{\text { peak }}{R M S}
$$

KuRTOSIS:Kurtosis is obtained from the fourth order central moment(moment about the mean) of amplitude probability distribution and is defined as-

$$
\text { - Kurtosis }=\frac{\frac{1}{N} \sum_{i=1}^{N}(x(i)-x)^{4}}{(R M S)^{4}}
$$

IMPULSE FACTOR:The impulse factor which is also found to be an indicator of bearing faults is defined as the ratio of the peak value to mean value of the time signal.

- Impulsefactor $=\frac{\text { peak }}{\frac{1}{N} \sum_{i=1}^{N}|x(i)|}$

SHAPE FACTOR: Shape factor is defined as the ratio of the RMS to mean value.It represents changes under unbalance and misalignment. 
- $\quad$ Shapefactor $=\frac{R M S}{\frac{1}{N} \sum_{i=1}^{N}|x(i)|}$

Where $\bar{x}$ denotes the mean value of the discrete time signal $\mathrm{x}(\mathrm{t})$ having $\mathrm{N}$ data points. Other time domain parameters are the clearance factor, impulse factor and shape factor. These three non-dimensional vibration amplitude parameters were found to be useful under simulation conditions using a Gaussian probability density function model of fatigue spalling. The clearance and impulse factors were the most useful with the clearance factor being the most sensitive and generally robust for detection of incipient fatigue spalling.

P.D.McFadden used the time domain technique in his Interpolation techniques for time domain averagingvibration of gear, Mechanical System and signal Processing(1989),Examination of a technique for the early detection of failure in gears by signal processing of the time domain average of the meshing vibration(1987) [6].

K.Umezawa.T.Ajima and H.Houjoh in their work "An acoustic method to predict tooth surface failure of in service gears" used the time domain technique to predict the failure analysis of gear mechanism [7].

\subsection{Frequency Domain Technique}

Frequency domain is the domain for analysis of mathematical functions or signals with respect to frequency, rather than time. To analyse the frequency distribution of vibration waveforms it is necessary to transform the time domain signal into frequency domain. Fourier transform is used to process the vibration signals obtained from time domain into the frequency domain in the form of Fast Fourier Transform [FFT].

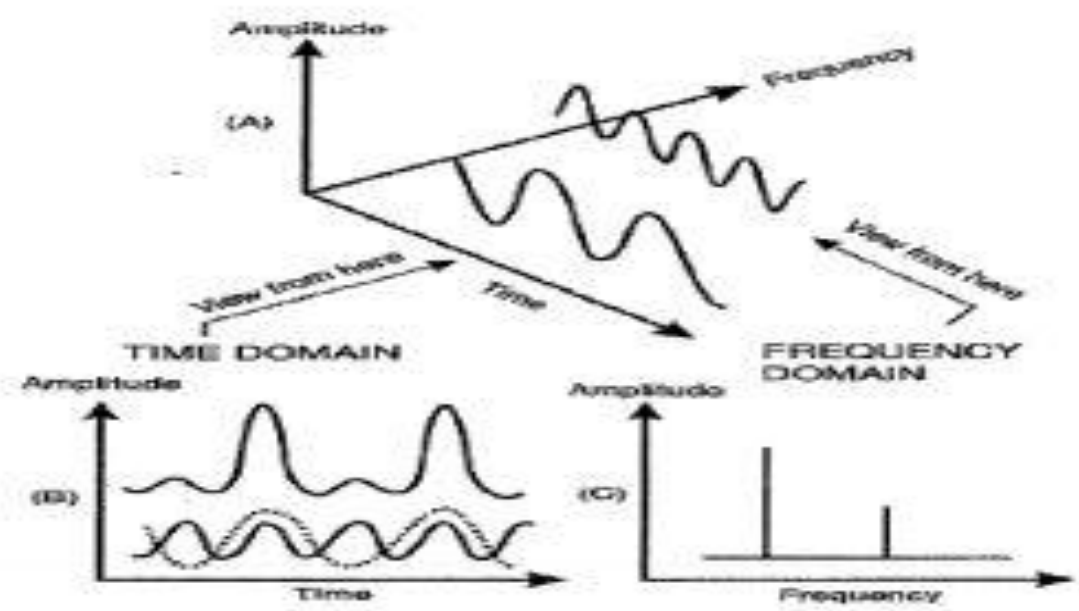

Fig 5: Fourier transformation to time domain signal to frequency domain.

The principal advantage of the method is that the repetitive nature of the vibration signals is clearly displaced as peaks in the frequency spectrum at the frequency where the repetition takes place.A frequency domain representation can also include information on the phase shift that must be applied to each sinusoid in order to be able to recombine the frequency components to recover the original time signal.

If adiscrete time signal $\mathrm{x}(\mathrm{t})$ represents a sampled periodic function with period $\mathrm{T}$, the Fourier series expansion of $\mathrm{x}(\mathrm{t})$ can be obtained from the Fourier integral

$$
X(f)=\frac{1}{T} \int_{-T / 2}^{T / 2} x(t) e^{\wedge-i 2 \pi f t} d t
$$

Where $f$ represents discrete equally spaced frequencies being multiples of the reciprocal of the period T.The power spectrum $\mathrm{P}(\mathrm{f})$ can be defined as the magnitude or power obtained from the Fourier integral and can beobtained by:

$P(f)=E\left[X(f) X^{*}(f)\right]$

Where*represents complex conjugation and E [ ] represents the expected value.

Spectra Analysis provides frequency domain amplitude in terms of displacement, velocity, acceleration or phase. Auto-spectrum or power spectrum is a similarity measurement in the frequency domain; the 
amplitudes are expressed as the square of their magnitudes. Cross-spectrum is a similarity measurement of two functions in the frequency domain.

Cepstrum analysis proves to be advantageous as the periodic harmonics, which are commonly experienced in gearboxes, can be detected even when they are covered within a high background noise. Cepstrum analysis may not work when more than two signals have been convolved in time domain.

CheeKeongTan,PhilIrving,DavidMba studied the prognosis of gear life using $\mathrm{AE}$ technique by allowing natural pitting of spur gears.In their test they monitored vibration, $\mathrm{AE}$ and SOA throughout the test period to correlate it to the natural degradation of the gear box. They found that based on the analysis of rms levels $\mathrm{AE}$ is more sensitive than the either of the rest[8].

Tim Toutountzakis,CheeKeong Tan and David Mba applied the AE technique to seeded gear fault detection and investigated the effectiveness of $\mathrm{AE}$ for gear defect identification and also its difficulties[9].

Bispectrumis yet another technique which is mainly employed to detect gear fault and have an edge over Fourier transform as cited by Zheng et al.., 2002that fourier transform is based on the assumption that the vibration signals are stationary. This bispectrum analysis has been proven to be effective in this situation. It can capture characteristic frequency, identify the phasic information and express nonlinear advantages (Huang et al., 2006). Liu et al. (2008) used the bispectrum and 1(1/2)-dimension spectrum to diagnose a gearbox with various pitting faults. They concluded that bispectral analysis was very sensitive to the gear faults.

Fu et al. (2009) applied the bispectrum technique to the gear wear fault detection and identification and Zhang (2006) to the gear crack fault diagnosis. Their studies showed that the bispectrum could be used as a diagnostic tool for gear faults detection.

\subsection{TIME FREQUENCY TECHNIQUe}

Time Frequency analysis is the technique in which both frequency and time domains are studied to represent a signal processing. Instead of the conventional method of studying a 1-dimensional analysis and some transform, time frequency analysis studies a 2-dimensional signal whose function is a 2-dimensional real plane. The most basic form of time-frequency analysis is the short-time Fourier transform (STFT), but more sophisticated techniques have been developed, notably wavelets.

The Short Time Fourier Transform of a signal $x(t)$ using a window function $g(t)$ is defined as follows-

$$
\operatorname{STFT}(f, s)=\int_{-\infty}^{\infty} x(t) g(t-s) e^{\wedge-i 2 \pi f t} d t
$$

The wavelet families are:

Continuous wavelet Transform: A continuous wavelet transform (CWT) is used to divide a continuous-time function into wavelets. Unlike Fourier transform, the continuous wavelet transform possesses the ability to construct a time-frequency representation of a signal that offers very good time and frequency localization. In mathematics, the continuous wavelet transform of a continuous, square-integrable function $x(t)$ at a scale $a>0$ and translational value $b \in \mathbb{R}$ is expressed by the following integral

$$
X_{w}(a, b)=\frac{1}{\sqrt{|a|}} \int_{-\infty}^{\infty} x(t) \psi^{*}\left(\frac{t-b}{a}\right) d t
$$

Morlet WaVelet Transform:A continuous function in both the time domain and the frequency domain is called the mother wavelet. The main purpose of the mother wavelet is to provide a source function to generate the daughter wavelets which are simply the translated and scaled versions of the mother wavelet.

DiSCRETE WAVELET:In numerical analysis and functional analysis, a discrete wavelet transform (DWT) is any wavelet transform for which the wavelets are discretely sampled. As with other wavelet transforms, a key advantage it has over Fourier transforms is temporal resolution: it captures both frequency and location information (location in time). They are of two types:
(a)Haar wavelets
(b)Daubechies wavelets

M.Elforjani,D.Mba,A,Sire and A.Muhammad worked on the condition monitoring of key components in rotating machinery such as gear box with $\mathrm{AE}$ and made comparisons with vibration analysis. Their observations revealed that $\mathrm{AE}$ parameters such as r.m.s and energy are more reliable, robust and sensitive to the detection of faults and defects [10]. 
K.Umezawa,T.Ajima and H.Houjoh diagnosed the extent of pitting fatigue on the basis of a frequency fluctuating analyzing method. The results obtained by them are by pitting fatigue increased, the fluctuation become stronger and the F/V output increased. This increases correlates with the number of load cycles by using $\mathrm{AE}$ techniques [11].

\subsection{EnVelope Analysis (High FrequenCy ResonanCe TeChNique - HFRT)}

The technique is extensively used in vibration analysis for rolling element fault diagnosis. The technique utilizes the high frequency region of the frequencyspectrum. The interaction of the defect in the gear produces pulses of very short duration, whereas the defect strikes the rotation motion of the system. These pulses excite the natural frequency of the gear elements, resulting in the increase in the vibration energy at these high frequencies.The resonant frequencies can be calculated theoretically. Each gear element has a characteristic rotational frequency. With a defect on a particular bearing element, an increase in the vibration energy at this element rotational frequency may occur. This defect frequency can be calculated from the geometry of the bearing and element rotational speed.

This technique allows a small bandwidth in the high frequency region to be reproduced in lower frequency waveform. Other high frequency commercial analysis techniques include PeakVue, Spike Energy (gSE), Spectral Emission Energy (SEE) and the Shock Pulse Method (SPM).

\section{DATA MINING TECHNIQUES}

Data Mining, also called as data or knowledge discovery, is a process used in different manufacturing industries to analyze data from different views and derive the useful information from them. It is one of the many analytical tools for analyzing data yet the most efficient one. It helps the user to find correlation and patterns among variety of different fields in large relational databases. The use of data mining techniques began in the early 1990s and has paved its path ever since. Now a days data mining is used to extract useful data that finds useful in predictive maintenance, fault maintenance, quality assurance, scheduling, and decision support systems. Many signal processing techniques have been developed and applied for machine diagnosis in this area.Decision trees one of the most important techniques of data mining are tree shaped structures that represents set of decisions. Rules are generated from these decisions to classify the dataset. Specific decision tree methods include Classification and Regression Trees (CART) and Chi Square Automatic Interaction Detection (CHAID).For classification of dataset CART and CHAID are used. Rules can be established for a new dataset which would predict which records will have which outcome. A two way splits is created by the CART to segments a dataset while multi way splits are created by CHAID to segment a dataset using square tests. CART typically requires less data preparation than CHAID.He et al. (2011) used decision tree method to identify fault in of mean shifts in bivariate processes inrealtime[12]. Artificial neural networks and Fuzzy Logic Systems establish a nonlinear I/O relationship which promotes mapping the input of the system to its output.

The complex nonlinear relation is reconstructed using simple functions such as sigmoid or radial which could be adjusted by means of appropriate parameter and synaptic weight.Artificial neural network is considered to be a star technique as it can readjust as per the requirement automatically during the training phase based on the I/O data.On the contrary in FLSs, the I/O spaces gets partitioned into several typically overlapping areas whose shapes can be determined by assigned memberships functions and its mapping relation is governed by simple IF-THEN rule. The major advantages of FLSs are that it can handle imprecise data, its physical transparency and the interpretability offered by it. When the inputs are the decision variables and the I/O space is seen as a searching space and the outputs are the performance indicator of the search problem, GAs proves a versatile tool for evaluating a best input solution.Kasabov,Marzi,Markou and Singh used many different methods based on artificial neural network which were developed for online surveillance, fault detection and learning abilities[13] [14] [15]. Wang (2002), used Artificial neural network (ANN), Fuzzy Logic System (FLS), Genetic Algorithms (GA) and Hybrid Computational Intelligence (HCI) in fault diagnosis[16]. Zhenyou Zhang et al., used WPD, FFT and BP neural network for fault diagnosis and prognosis. The analysis was focused on the vibration signals collected from sensors mounted on the critical component. The features extracted from the frequency domain were used to train ANN. Trained ANN then predicted the degradation and identified the faults of component[17]. Saravanan et al. 2008 used support vector machine (SVM) for fault diagnosis of spur bevel gear box and this is a popular machine learning application due to its high accuracy and good generalization capabilities[18]. Huang et al.(2008) used probability based Bayesian network methods to identify vehicle fault which can be used to diagnose single-fault and multi-fault[19]. Zhixiong Li et al, usedthe back propagation neural network (BPNN) and the radial-basis function neural network (RBFNN) to identify the states of the gearbox.The numeric and experimental test results showed that the ANN classification method has achieved high detection accuracy[20]. Weixiang Sun, Jin Chen and Jiaqing Li used data mining technology after conventional fault testing.They proposed a new method based on C4.5 decision tree and principal component 
analysis(PCA).It was found that compared to BPNN C4.5 extracts knowledge quickly from the testing and is even superior to neural networks[21].

There are several other techniques like nearest neighbor method that classifies each record in a dataset based on a combination of the classes of the $k$ record(s) most similar to it in a historical dataset. Data visualization is a visual interpretation of complex relationship in multidimensional data. It makes use of graphics tools to illustrate data relationships. Yaguo Lei and Ming J. Zuo used a two stage feature selection and weighting technique(TFSWT) via Eucledian distance evaluation technique(EDET) to select sensitive features and remove fault unrelated features. They used a weighted K nearest neighbor(WKNN) classification algorithm to identify the egar crack levels. The resolutionshowed that this method had higher identification accuracy[22].

\section{CONCLUSION:}

In this review paper the various types of defects related to gear boxes have been discussed. It was clearly stated that vibration signature analysis and acoustic emission are two very efficient techniques for early fault detection. Vibration signature analysis is used for detect major defects whereas acoustic analysis is used to detect very minute cracks occurring in the gear box. Various signal processing techniques were discussed. The importance of wavelet transformation has also been mentioned which is now a day's widely used for feature extraction as waves associated to the gear boxes are non stationary nature. Various other data mining techniques have been mentioned for a clear understanding of feature extraction. Relevant research work carried out related to each have been discussed in brief.

\section{REFERENCES}

[1]. Assessment of Bearing Fault Detection Using Vibration Signal Analysis by Amit R. Bhende, GK Awari and SP Untawale

[2]. Sound and Vibration Measurement A/S,Bruel\&Kjaer

[3]. Vibration-based fault diagnosis of spur bevel gear box using fuzzy techniqueN. Saravanan, S. Cholairajan, K.I. Ramachandran

[4]. A comparison of Acoustic Emission and Vibration on Bearing Fault Detection by Xiaoqin Liu, Xing Wu, Chang Liu.Faculty of Mechanical and Electrical Engineering,Kunming University of Science and Technology,Kunming, China.

[5]. Practical Machinery Vibration Analysis and Predictive Maintenance,(Scheffer and Gridhar)

[6]. Interpolation techniques for time domain averaging vibration of gear, Mechanical System and signal Processing(1989),Examination of a technique for the early detection of failure in gears by signal processing of the time domain average of the meshing vibration(1987) by P.D.McFadden

[7]. An acoustic method to predict tooth surface failure of in service gears by K.Umezawa.T.Ajima and H.Houjoh.

[8]. A comparative experimental study on the diagnostic and prognostic of acoustic emission,vibration and spectrometric oil analysis(2005) by CheeKeongTan ,Phil Irving,DavidMba.

[9]. Application of Acoustic Emission to seeded gear fault detection (2005)by Tim Toutountzakis,CheeKeong Tan and David Mba.

[10]. Condition monitoring of worm gears (2012) by M.Elforjani,D.Mba,A,Sire and A.Muhammad.

[11]. An acoustic method to predict tooth surface failure of in servicegears(1982) by K.Umezawa,T.Ajima and H.Houjoh.

[12]. He, S., He, Z.,\& Wang, G. (2011). Online monitoring and fault identification of mean shifts in bivariate processes using decision tree learning techniques. Journal of Intelligent Manufacturing, 1-10. doi:10.1007/s10845-011-0533-5.

[13]. Kasabov, N. (2001). Evolving fuzzy neural networks for supervised/ unsupervised online knowledge-based learning.IEEE Transactions on Systems, Man, and Cybernetics, Part B: Cybernetics, 31, 902-918.

[14]. Markou, M., \& Singh, S. (2003). Novelty detection: A review-part 2:Neural network based approaches. Signal Processing, 83, 2499-2521.

[15]. Marzi, H. (2004). Real-time fault detection and isolation in industrial machines using learning vector quantization. Proceedings of the Institution of Mechanical Engineers, Part B: Journal of Engineering Manufacture, 218, 949-959.

[16]. Wang, K. (2002). Intelligent condition monitoring and diagnosis systems.

[17]. Zhixiong Li, Xinping Yan, Chengqing Yuan, Jiangbin Zhao and ZhongxiaoPeng. Fault Detection and Diagnosis of a Gearbox in Marine Propulsion Systems Using Bispectrum Analysis and Artificial Neural Networks.Article ID: 1671-9433(2011)01-0017-08

[18]. Saravanan, N. K., Sidddabattuni, V. N. S., \&Ramachandran, K. I. (2008). A comparative study on classification of features by SVM and PSVM extracted using Morlet wavelet for fault diagnosis of spur bevel gearbox. Expert System with Applications, 35, 13511366

[19]. Huang, Y., Mcmurran, R., Dhadyalla, G., \& Jones, R. P. (2008). Probabilitybased vehicle fault diagnosis: Bayesian networkmeth od. Journal of Intelligent Manufacturing, 19(3), 301-311.

[20]. Zhixiong Li, Xinping Yan, Chengqing Yuan, Jiangbin Zhao and ZhongxiaoPeng. Fault Detectionand Diagnosis of a Gearbox in Marine Propulsion Systems Using Bispectrum Analysis and Artificial Neural Networks.Article ID: 1671-9433(2011)01-0017-08

[21]. Decision tree and PCA-based fault diagnosis of rotating machinery(2006) by Weixiang Sun, Jin Chen, Jiaqing Li.

[22]. Gear crack level identification based on weighted K nearest neighbor classification algorithm(2009) by YaguoLei, MingJ.Zuo 